\title{
Helical carbon nanotubes alter f-actin and arp2 distribution in macrophages and inhibit pseudomonas aeruginosa phagocytosis in an intracellular accumulation-dependent manner
}

\begin{abstract}
The similarity of carbon nanotubes (CNTs) to the shape and size of asbestos fibers has raised concern over their potential pulmonary toxicity in occupational and environmental settings. Studies have demonstrated pulmonary toxicity associated with exposure to CNTs, including modulation of the pulmonary immune system and impaired phagocytosis. However, mechanisms underlying this inhibition have not been fully elucidated. In this study, intracellular accumulation of helical CNTs (HCNTs) and inhibition of Pseudomonas aeruginosa internalization were measured in RAW 264.7 and alveolar macrophages. Distribution and co localization of F-actin and actin-related-protein 2 (Arp2) - key proteins in the formation of phagosomes - by HCNTs, were examined using fluorescent microscopy, and inhibition of F-actin polymerization was quantified. Our results indicated that inhibition of $P$. aeruginosa internalization by RAW 264.7 and alveolar macrophages was directly related to the amount of intracellular accumulation of HCNTs. Additionally; HCNTs inhibited F-actin polymerization in vitro, and disrupted normal F-actin and Arp2 distribution in RAW 264.7 macrophages. Disruption of F-actin polymerization, and F-actin and Arp2 distribution, may explain how HCNTs interfere with the phagocytosis of pathogens by macrophages. This information can also contribute to evaluating the health risk of individuals exposed to potentially high levels of CNTs.
\end{abstract}

Keywords: Helical carbon nanotubes, Macrophages, Phagocytosis, F-actin, Arp2
Volume 2 Issue I - 2015

\section{Brent EWalling, Zhizhou Kuang, Yonghua Hao, Constantine Gastouniotis, Gee W Lau Department of Pathobiology, University of Illinois at Urbana- Champaign, USA}

Correspondence: Gee W Lau, Department of Pathobiology, University of Illinois at Urbana-Champaign, Urbana, IL, 61802, USA, Tel (I) 217-333-5077 Email geelau@illinos.edu

Received: January 19,2015 | Published:January 27, 2015
Abbreviations:Arp2, Actin-Related-Protein 2; CNTs, Carbon Nanotubes; HCNTs, Helical Carbon Nanotubes; SWCNTS, Single Walled Carbon Nanotubes; MWCNTS, Multi Walled Carbon Nanotubes; DMEM , Dulbecco's Modified Eagle Medium; BAL, Broncho Alveolar Lavage; RPMI, Roswell Park Memorial Institute; DAPI, 4',6-Diamidino-2-Phenylindole; MOI, Multiplicity of Infection; CFU, Colony Forming Units

\section{Introduction}

Production of carbon nanotubes (CNTs) for commercial use has rapidly expanded due to their successful utilization for applications across multiple fields, including polymer production and electronics. The production methods and ubiquitous presence have led toxicologists to examine the potential toxicity of CNTs in occupational and environmental settings, with a strong concern for pulmonary injuries. ${ }^{1}$ Recent studies show that CNTs cause pulmonary granulomas and fibrosis in mice..$^{2-4}$ Additionally, CNTs modulate the pulmonary innate immune response, increasing its susceptibility to infections..$^{5-7}$ This impaired pulmonary immunity has been, in part, attributed to inhibition of pathogen phagocytosis by macrophages. ${ }^{7,8}$ Furthermore, CNTs can interfere with the phagocytosis of latex beads and apoptotic cells. ${ }^{9-11}$ However, the mechanisms underlying phagocytic inhibition remain unclear.

Alveolar macrophages play an important role in defending the lung against infection by microbial pathogens. They act as sentinels in the alveolar spaces and, upon encountering pathogens, macrophages phagocytize the microbes and activate an array of downstream signals. ${ }^{12}$ Successful opsonic and non-opsonic phagocytosis of pathogens depends on the assembly of the cytoskeletal protein F-actin to form phagosomes. ${ }^{13}$ Coordination of proper assembly of actin in cells relies on a protein complex, which includes the proteins Arp2 (actin-relatedprotein 2) and Arp3, which serves as sites of actin nucleation and branching. ${ }^{14}$ Interruption of F-actin assembly dramatically alters the cytoskeleton and interferes with proper cell function. This has been demonstrated using mycotoxin cytochalasin $\mathrm{D}$, which disrupts normal cell architecture and inhibits phagocytosis by binding directly to F-actin monomers, inhibiting polymerization and depolymerization. ${ }^{15}$ Importantly, inhibition of Arp2/3 recruitment prevents internalization of latex beads in COS cells, implicating the necessary role of this protein complex in the formation of phagosomes. ${ }^{16}$

Previous studies have demonstrated that internalization of ultrafine particles, including TiO2 by J774A.1 macrophages, caused cytoskeletal dysfunction and altered phagosome motility. ${ }^{17}$ Also, TiO2 exposure caused myofibrillar structure disorganization in cultured neonatal rat ventricular myocytes. ${ }^{18}$ Moreover, internalization of CNTs by cells may disrupt the cytoskeleton. Kaiser et al. ${ }^{19}$ reported alterations of F-actin filaments in A549 alveolar epithelial cells and mesothelioma cells following exposure to raw single walled CNTs (SWCNTs) but not purified SWCNTs, suggesting that the effect was due to presence of catalytic metals or amorphous carbon associated with the production of nanotubes. However, Holt et al. ${ }^{20}$ was able to demonstrate that purified SWCNTs could cause actin-related cell division defects, and SWCNTs can alter F-actin structures and focal adhesion complexes in HeLa cells. Another study showed that SWCNTs altered the distribution of the actin cytoskeleton and clathrin in mast cells. ${ }^{21}$ Furthermore, a separate study showed that multi walled CNTs (MWCNTs) altered both F-actin and tubulin expression and distribution in human bronchial epithelial cells. ${ }^{22}$ Collectively, these studies suggest that CNT exposure results in 
alteration of F-actin distribution and/or expression but this may be dependent on specific types of purified CNTs and cell lineages used. Our work was undertaken to examine how a type of MWCNTs, helical CNTs (HCNTs), affect Arp2 and F-actin distribution in immortalized macrophages. Additionally, we examined whether the accumulation of HCNTs that disrupted Arp2/3-F-actin in macrophages also inhibited phagocytosis of $P$. aeruginosa.

\section{Materials and methods}

\section{Chemicals and cell lines}

Chemicals were purchased from Sigma-Aldrich, unless stated otherwise. Actistain-555 was purchased from Cytoskeleton, Inc. Prolong Gold mounting medium was purchased from Invitrogen. The murine RAW 264.7 macrophages were purchased from ATCC (\#TIB-71). RAW 264.7 macrophages were maintained in Dulbecco's modified eagle medium (DMEM) supplemented with $10 \%$ fetal bovine serum, penicillin, and streptomycin until $70-80 \%$ confluence at $37^{\circ} \mathrm{C}$ in 5\% CO2. Alveolar macrophages from untreated CD-1 mice were harvested by broncho alveolar lavage (BAL), pooled, resuspended in Roswell Park Memorial Institute (RPMI) medium, and plated in wells containing autoclaved glass cover slips at a density of $5 \times 105$ cells per well for 2 hours at $37^{\circ} \mathrm{C}$ in $5 \% \mathrm{CO} 2$ to allow attachment.

\section{Preparation of HCNTs}

HCNTs (Cheap Tubes Inc.) were suspended to $1 \mathrm{mg} / \mathrm{ml}$ in DMEM without phenol (Gibco) with dispersal media $(0.01 \%$ Tween- 80 in PBS), vortexed, sonicated on ice, and diluted to desired concentrations. Physical characteristics of the HCNTs were previously determined with TEM, SEM, DLS, and Raman spectroscopy. ${ }^{8}$

\section{Examination of F-actin and Arp2 distribution}

RAW 264.7 macrophages were placed on glass cover slips at a density of 1 X105 cells/well and allowed to adhere overnight. The following day, macrophages were washed and exposed to HCNTs at a concentration of 0,10 , and $100 \mu \mathrm{g} / \mathrm{ml}$ for 24 hours, similar to low and high levels of in vitro exposure from previously published studies. ${ }^{7,11}$ Cells were then washed with PBS and fixed with $4 \%$ paraformaldehyde for 1 hour at room temperature. Fixed cells were washed and permeabilized with $0.1 \%$ Triton X-100 for 30 minutes at room temperature. Following permeabilization and washing, cells were blocked with 5\% BSA incubated for one hour at room temperature followed by incubation with 1:100 anti-Arp2 antibody (Abcam, Inc.) for 2 hours. After washing with PBS, cells were incubated in the dark with $100 \mathrm{nM}$ phalloidin (a phallotoxin which binds at the interface between F-actin subunits) conjugated to the fluorescent dye rhodamine (Acti-stain 555 phalloidin, Cytoskeleton, Inc.) and 488-labeled goat anti-rabbit antibody for 30 minutes. Cells were then washed and coverslips were mounted onto glass slides with an antifade mounting medium containing 4',6-diamidino-2phenylindole (DAPI) (Prolong Gold, Invitrogen).

Harvested murine alveolar macrophages (see below) were plated in wells with glass coverslips at a density of 1X105 cells/well and allowed to adhere over 2 hours. The macrophages were then washed and exposed to HCNTs at a concentration of 0,10 , and $100 \mu \mathrm{g} / \mathrm{ml}$ for 1 hour. Cells were then washed, fixed, permeabilized, stained, and mounted as described above. Internalization of HCNTs and localization of F-actin and Arp2 was determined on an Olympus Fluoview confocal system using a Melles Griot Krypton laser with a 100X oil objective and proprietary Olympus "Fluoview" software $(\mathrm{FV} 300 \vee 5)$.
To measure the accumulation of HCNTs within the intracellular space of macrophages, TIFF images obtained from confocal microscopic examination of macrophages were analyzed using the Adobe Photoshop software. Individual cells were randomly selected and pixel intensity was examined using a histogram, which assigned a numeric value for the brightness of individual pixels from 0 (black) to 255 (white). Areas of unencumbered macrophages and extracellular space were used to establish the threshold for the darkest pixels not associated with HCNTs. Macrophages with HCNTs were then analyzed and the percent of pixels with an intensity value below the threshold set by the unencumbered macrophages was obtained. HCNT-laden macrophages were categorized based on percent pixels below the set threshold (i.e. $1-20 \%, 21-40 \%$, etc.) and enumerated for the presence of PAO1-GFP in the cytoplasm.

Analysis of mean fluorescence for both F-actin and Arp2 was performed using the Image $\mathrm{J}$ software by examining the stack of images (slices) collected by confocal microscopy. For each cell, the bottom and the top slice were visualized, the distance between them was measured and nine equidistant slices were determined, giving a total of 11 slices including the bottom (slice 1), middle (slice 6), and top (slice 11). For each slice, the cell border was traced and both the mean F-actin (red) and Arp2 (green) fluorescent intensity was measured. Slice 6 was selected to represent the middle of the cell for subsequent analysis. A line from cell edge to the opposite cell edge was drawn to both include the nucleus (long axis) and exclude the nucleus (short axis). Fluorescence intensity for F-actin and Arp2 was measured along the line and 51 equidistant points, from edge to edge, were used for analysis.

\section{In vitro F-actin polymerization assays}

The effects of HCNTs on F-actin polymerization were quantified using the Actin Polymerization Biochem Kit (Cytoskeleton, Inc.) following the manufacturer's instructions. Briefly, RAW 264.7 macrophages were allowed to adhere overnight. Depolymerized F-actin and HCNTs (at concentrations of 0,10 , or $100 \mu \mathrm{g} / \mathrm{ml}$ ) were added to the wells prior to the addition of actin polymerization buffer. Fluorescence was measured until the signal plateaued at approximately 70 minutes.

\section{In vitro $\mathbf{P}$. aeruginosa internalization assays}

Internalization of the GFP-expressing $P$. aeruginosa strain PAO1GFP by HCNT-exposed RAW 264.7 macrophages was evaluated using confocal microscopy. Initially, 1X106 RAW 264.7 macrophages were placed on 6-well tissue culture plates overnight. Macrophages were washed and HCNTs were added at a concentration of 0,10 or $100 \mu \mathrm{g} /$ $\mathrm{ml}$ for 24 hours. Macrophages were washed to remove non-internalized nanotubes and incubated with PAO1-GFP at a multiplicity of infection (MOI) of 10:1. After 1 hour, macrophages were washed to remove extracellular PAO1-GFP, and then fixed with $4 \%$ paraformaldehyde for 1 hour at room temperature. Coverslips were mounted on glass slides with per mount and sealed with clear nail polish. Macrophages were subsequently examined and enumerated for the presence of internalized bacteria by confocal fluorescence microscopy.

\section{HCNT exposure and P. aeruginosa infection in mice}

Animal studies were performed with approval by the Institutional Animal Care and Use Committee at the University of Illinois at Urbana-Champaign. Six-week old wild-type CD-1 mice (Charles River Laboratories) were housed in positively ventilated micro isolator cages with automatic recirculating water, located in a room with laminar, high efficiency particle accumulation-filtered air. The 
animals received autoclaved food, water, and bedding. Mice were intranasally inoculated with $50 \mu \mathrm{g}$ of HCNTs in $50 \mu \mathrm{l}$ of dispersal media or equivalent volume of dispersal media twice/week for 3 weeks, a protocol similar to those used in previously published studies exploring the impact of CNTs on the pulmonary immune system. ${ }^{23,24}$ Under this protocol, the calculated equivalent human exposure would be obtained in 8.4 years, 1.1 years, and 2.7 months when exposed to 53,400 , and $2000 \mu \mathrm{g} / \mathrm{m} 3$, respectively, which represent airborne concentrations that have been measured from facilities utilizing different and variably rigorous controls to limit airborne exposure. ${ }^{8}$ Three days after the final HCNT dose, mice were intranasally inoculated with 1X107 colony forming units (CFU) of PAO1-GFP. After 1 hour, mouse lungs were lavaged for cell collection and confocal microscopy to examine internalized bacteria. BAL was performed as previously described. ${ }^{25,26}$ Briefly, the trachea was exposed and $1.0 \mathrm{ml}$ cold PBS was injected and removed from the lungs through an 18 gauge needle. This first aliquot was placed on ice. Mouse lungs were BAL three more times with $1.0 \mathrm{ml}$ and pooled separately. The first aliquot was centrifuged at $500 \mathrm{~g}$ for 5 minutes and the supernatant was removed and preserved at $-80^{\circ} \mathrm{C}$. The cells from the first aliquot were resuspended in PBS and pooled with the additional $3 \mathrm{ml}$ of BALF. Cells were concentrated on glass slides by cytospin and fixed with $4 \%$ paraformaldehyde overnight at $4^{\circ} \mathrm{C}$. Internalization of PAO1-GFP was determined by evaluating at least 200 macrophages from each mouse by confocal microscopy.

\section{Statistical analysis}

Normality of the data was evaluated using the Anderson-Darling normality test with rejection of normality when $p$-value $<0.05$. For comparing the means of 2 groups, the Student's t-test was used. For comparing the means of groups of three or more, data were analyzed for statistical significance by ANOVA followed by Tukey's tests for comparison between the means.

\section{Results}

Initially, we examined whether the presence of HCNTs in the intracellular space impair phagocytosis. Additionally, we were curious whether exposure to different concentrations of HCNTs led to different levels of intracellular accumulation and how this might alter the uptake of $P$. aeruginosa. RAW 264.7 macrophages were exposed to different concentrations of HCNTs for 24 hours before exposure to a GFP expressing $P$. aeruginosa strain PAO1-GFP. Confocal microscopy showed that pre-exposure to both low concentration (10 $\mu \mathrm{g} / \mathrm{ml})$ and high concentration $(100 \mu \mathrm{g} / \mathrm{ml})$ of HCNTs significantly inhibited internalization of PAO1-GFP by RAW 264.7 macrophages (Figure 1a \& 1b). Using Photoshop software, we quantified the percentage of intracellular cytoplasmic space occupied by HCNTs and correlated obtained data to observed internalization of PAO1. We used a simplified method of quantifying intracellular particle accumulation, which differed from approach published by other investigators. ${ }^{27,28}$ For example, Muhlfeld et al. ${ }^{27}$ developed a relative deposition index which examined distribution of nanoparticles by size in cellular compartments but acknowledged its limitation in the extrapolation of quantification. Tian et al. ${ }^{28}$ developed a "max-flat assay" using confocal microscopy which could accurately quantify the uptake of fluorescent polystyrene beads by counting each bead. However, because of the variety in sizes and shapes of the HCNTs, as well as some aggregation, counting individual HCNTs was not possible. Instead, we exploited the distinct contrast between the HCNTs and surrounding intracellular space, which allowed for accurate intracellular quantification as a percentage of intracellular space.

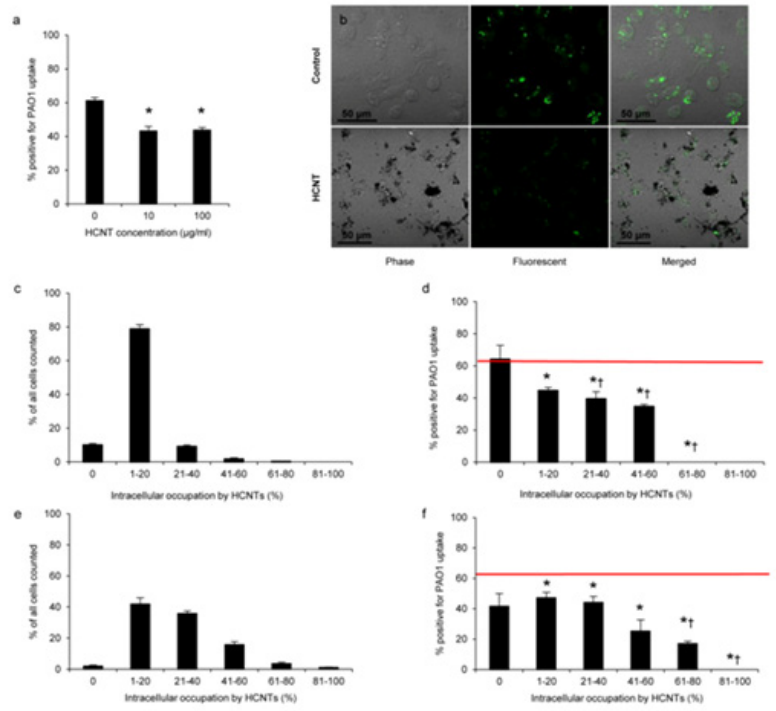

Figure I HCNTs inhibit internalization of $P$. aeruginosa by RAW 264.7 macrophages.

1a: Quantification of the number of macrophages that internalized PAO1-GFP bacteria following 24hours of exposure to 10 or $100 \mu \mathrm{g} / \mathrm{ml} \mathrm{HCNT}$. All experiments were performed in triplicate and independently repeated 3 times with similar results. 1b: Representative phase, fluorescent, and merged micrographs of control and HCNT treated cells after exposure to PAO1-GFP. 1c \& 1e: Macrophages exposed to HCNTs were grouped based on the percentage of the intracellular space occupied by HCNTs. The majority of macrophages exposed to HCNTs at a concentration of $10 \mu \mathrm{g} / \mathrm{ml}$ have accumulated enough HCNTs to occupy $1-20 \%$ of the intracellular space (c) while increased intracellular burdens were observed with macrophages exposed to HCNTs at a concentration of $100 \mu \mathrm{g} / \mathrm{ml}$ (e). 1d \& 1f: The percentage of PAO1-GFP positive macrophages in relation to increased intracellular HCNT accumulation (d). Red horizontal line indicates the percentage of PAO1-GFP positive macrophages not exposed to HCNTs run in parallel.

${ }^{*} \mathrm{p}<0.05$ (versus unexposed macrophages); $\dagger \mathrm{p}<0.05$ (compared to HCNT-exposed macrophages with no evidence of HCNT accumulation). Error bars indicate standard error of the mean.

\section{Time and concentration dependent intracellular accumulation of HCNTs by RAW 264.7 macrophages and the effect on P. aeruginosa internalization}

At a concentration of $10 \mu \mathrm{g} / \mathrm{ml}, 79 \%$ of RAW 264.7 cells had HCNTs occupying $1-20 \%$ of the intracellular space, while $10 \%$ of the cells showed $21-40 \%$ intracellular accumulation. About $2 \%$ of the cells showed $41-60 \%$ intracellular HCNT accumulation and $0.3 \%$ showed $61-80 \%$ accumulation. Finally, approximately $10 \%$ of macrophages showed no intracellular accumulation of HCNTs (Figure 1c). When exposed to $100 \mu \mathrm{g} / \mathrm{ml}$, the 264.7 RAW macrophages markedly increased their intracellular accumulation of HCNTs. The percentage of the cells showing a $21-40 \%$ intracellular HCNT accumulation increased to $36 \%$. The percentage of cells with $41-60 \%$ accumulation increased to $15 \%$ of the counted population whereas the percentage of cells with $61-80 \%$ accumulation increased to $\sim 3.5 \%$ of the total cells counted. Finally, a small number $(\sim 1 \%)$ of cells had accumulated enough HCNTs to fill $>80 \%$ of the intracellular 
space. On the other hand, the proportion of macrophages that had accumulated HCNTs occupying $1-20 \%$ of the intracellular space declined to $\sim 42 \%$ and the percentage of macrophages, which did not show any evidence of HCNT accumulation, reduced to $\sim 2 \%$ of the population counted (Figure 1e). Thus, for the same exposure time, intracellular accumulation of HCNTs was greater when exposed to a higher starting concentration of HCNTs. When compared against unexposed control RAW 264.7 macrophages, increasing intracellular accumulation of HCNTs was associated with a decrease in the uptake of PAO1-GFP (Figure 1d \& 1f). This was observed in macrophages exposed to both low and high concentrations of HCNTs. Additionally, as the intracellular HCNT burden increased, the percentage of PAO1GFP positive macrophages decreased, with the effect being more pronounced at an intracellular HCNT burden $>40 \%$ regardless of the exposure concentration. Thus, macrophages that have internalized HCNTs are impaired in their ability to subsequently internalize PAO1.

When examining cohorts within a single exposure group, a similar pattern emerged. At an exposure concentration of $10 \mu \mathrm{g} /$ ml HCNTs, the percentage of PAO1-GFP positive macrophages identified as having accumulated no HCNTs was $\sim 64 \%$, which was not statistically different than the cohort which had accumulated $1-20 \%$ HCNTs $(\sim 45 \%)$ within the intracellular space (Figure 1d). However, those macrophages that had accumulated HCNTs to occupy $>20 \%$ of their intracellular space were significantly inhibited compared to unencumbered macrophages. Interestingly, at $100 \mu \mathrm{g} /$ $\mathrm{ml}$, only those macrophages that had accumulated HCNTs $>40 \%$ of their intracellular space showed impaired internalization of PAO1GFP compared to unencumbered macrophages (Figure 1f). However, unencumbered macrophages in this exposure group represented $\sim 2 \%$ of all macrophages counted, which may have elevated the standard error, thus reducing statistical significance.

\section{Distribution of F-actin in RAW 264.7 macrophages following exposure and internalization of HCNTs}

Prior studies have demonstrated that CNTs may interfere with the cytoskeleton in HeLa, mast, and bronchial epithelial cells by forming bundle with F-actin filaments. ${ }^{20-22}$ Thus, we examined if exposure to HCNTs altered F-actin and Arp2 distribution in RAW 264.7 macrophages. Initial observations with confocal microscopy demonstrated F-actin accumulation (red fluorescence) along the base of both unexposed macrophages (Figure 2a) and HCNT-exposed macrophages (Figure 2c). Interestingly, cross sections of unexposed macrophages demonstrated abundant co-localization of F-actin and Arp2 (Figure 2b) whereas this was markedly reduced in the HCNTexposed macrophages (Figure 2d). Quantification of fluorescent intensity was then performed both across multiple slices from the base to the top of macrophages (Figure 2e), as well as across a long axis and short axis at the cell midline (Figure $2 \mathrm{~g} \& 2 \mathrm{~h}$ ). F-actin intensity was similar between exposed and unexposed cells both along the base and at the top. This result is expected as the base is the where cells attach to the coverslip, requiring abundant $\mathrm{F}$-actin polymerization. However, F-actin intensity was significantly lower within the lower $1 / 3$ to $1 / 2$ in those macrophages exposed to HCNTs at $100 \mu \mathrm{g} / \mathrm{ml}$ (Figure $2 \mathrm{f})$. Because of this observation, the pattern of F-actin distribution midway between the base and the top of the cells was selected and measured. To account for different sizes of macrophages observed as well as the presence of the nucleus, 51 equidistant measurements were taken across every cell in the long axis \{to include the nucleus, (Figure $2 \mathrm{~g}$ ) $\}$ and short axis \{excluding the nucleus, (Figure $2 \mathrm{~h}$ ) . F-actin expression patterns were similar for all groups, with the greatest intensity along the cell periphery and a noticeable decrease in the cytoplasm (short axis) and nucleus (long axis) (Figure 2i \& $2 j$ ). However, intra-cytoplasmic distribution and intensity, away from directly beneath the cell membrane, were significantly different in those macrophages exposed to HCNTs when compared to unexposed controls, most notably in macrophages exposed to $100 \mu \mathrm{g} / \mathrm{ml}$ (Figure $2 \mathrm{i} \& 2 \mathrm{j}$ ). Our results indicate that, at a high concentration of HCNTs, F-actin distribution is altered, particularly within the cytoplasm distant from the cell membrane. More importantly, central intra cytoplasmic

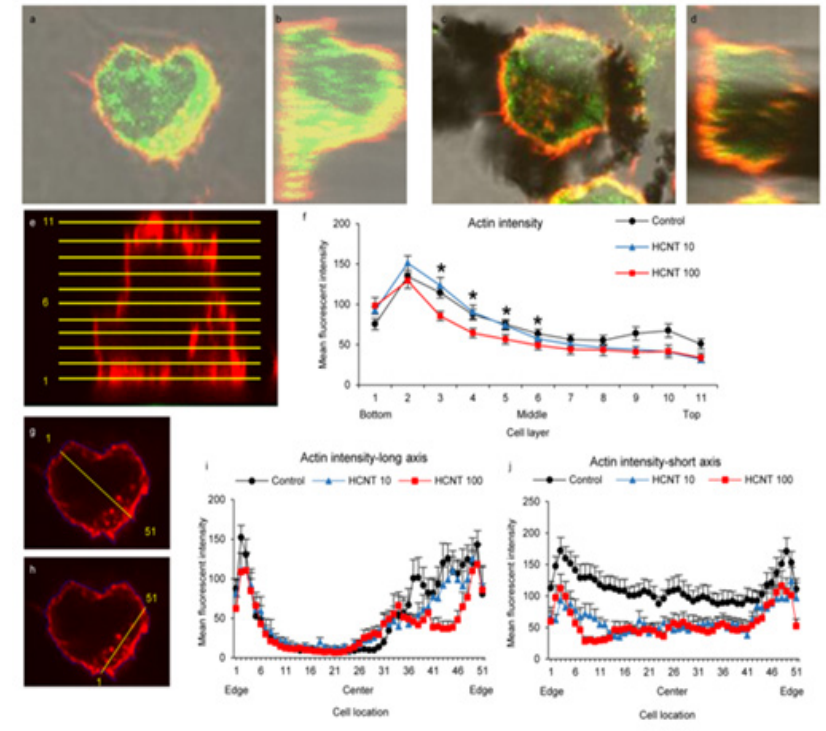

expression of $\mathrm{F}$-actin is decreased in macrophages exposed to HCNTs.

Figure 2 Exposure to HCNTs causes redistribution and impairs expression of F-actin in RAW 264.7 macrophages.

$2 a$ \& 2b: Confocal images of control RAW 264.7 macrophages with fluorescent tags for F-actin (red) and Arp2 (green) demonstrate individual and co localization (yellow) of both proteins. 2c \& 2d: Macrophages exposed to HCNTs show visibly decreased F-actin and Arp2 within the cytoplasm. $2 e$ \& 2f: F-actin fluorescence was measured across II slices for each macrophage. Slices were evenly distributed from the base to the top of the cells (e). Mean fluorescence intensity in the lower-middle area of macrophages (slices 2-6) was significantly decreased in macrophages exposed to $100 \mu \mathrm{g} / \mathrm{ml}$. $2 \mathrm{~g}-2 \mathrm{j}$ : Fluorescence was measured across the center of the cells at the mid line (slice 6). Both a long axis measurement $(\mathrm{g})$ which included the nucleus and a short axis measurement $(\mathrm{h})$ were used and equidistant $5 \mathrm{I}$ points from edge to edge were analyzed. Measurements across the cells showed the highest intensity along the cell membranes. Macrophages exposed to $100 \mu g /$ $\mathrm{ml}$ showed decreased fluorescence intensity in the mid cytoplasmic areas both along the long and short axis (i,j).

${ }^{*} \mathrm{p}<0.05$ (versus unexposed macrophages).

\section{HCNTs inhibit F-actin polymerization}

An F-actin polymerization assay was performed in which pyrene conjugated G-actin emits a fluorescent signal upon conjugation to F-actin following the addition of actin polymerization buffer. ${ }^{29}$ The presence of different concentrations of HCNTs alone did not result in any polymerization of F-actin (Figure 3). Following the addition of actin polymerization buffer at 20 minutes, $\mathrm{F}$-actin polymerization was rapid in both the untreated and HCNT treated wells. However, HCNTs inhibited both the rate and total F-actin polymerization, which was significant at a concentration of $100 \mu \mathrm{g} / \mathrm{ml}$. 


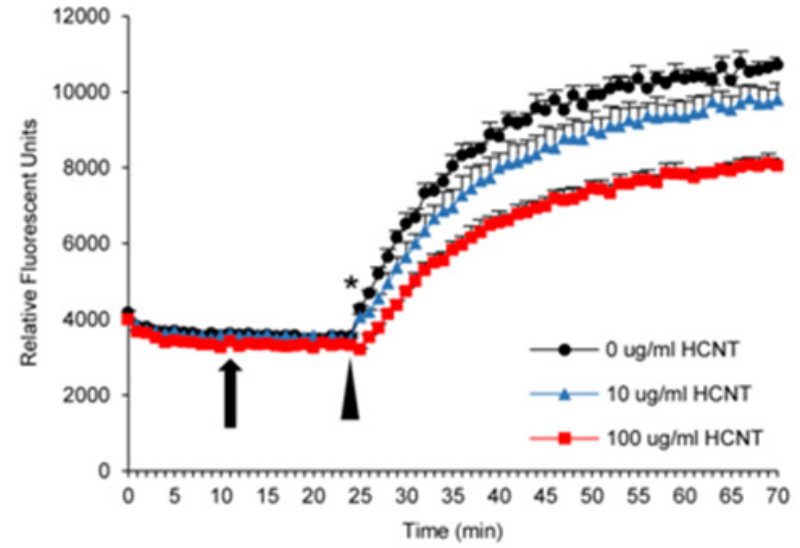

Figure 3 HCNTs inhibit F-actin polymerization. Depolymerized furanconjugated F-actin was incubated with HCNTs followed by the addition of polymerization buffer. The presence of HCNTs alone did not induce polymerization (arrow). Polymerization of F-actin occurred immediately following the addition of polymerization buffer in all groups (arrowhead). However, both concentrations of HCNTs inhibited polymerization, with 100 $\mu \mathrm{g} / \mathrm{ml}$ concentrations significantly inhibited at all time points. All groups were run in quadruplicate and the experiment was repeated once more with similar results.

${ }^{*} p<0.05$ compared to unexposed wells. Error bars indicate standard error of the mean.

\section{Arp2 levels and distribution in cells exposed to HCNTs}

Previous work has demonstrated that exposure to CNTs may alter the distribution of paxillin, which together with actin, are important in the formation of focal adhesion structures. ${ }^{20}$ Arp2 is one of the proteins of the Arp $2 / 3$ complex important in the formation of phagosomes. ${ }^{16}$ We examined the effect of HCNT exposure on both distribution and expression of Arp2. We applied the same methodology as described in Figure 2 for examining Arp2 distribution in control and HCNT-exposed RAW 264.7 macrophages. Mean Arp2 intensity was highest at the base of the cells for both unexposed and exposed macrophages (Figure $4 \mathrm{a} \& 4 \mathrm{~b}$ ) and there was no significant difference in Arp2 intensity between unexposed macrophages when compared to macrophages exposed to a $10 \mu \mathrm{g} / \mathrm{ml}$ concentration of HCNTs. However, mean fluorescence intensity across the middle of the cells was significantly lower in macrophages exposed to a $100 \mu \mathrm{g} /$ $\mathrm{ml}$ concentration of HCNTs compared to unexposed cells (Figure 4b). Arp2 expression at the middle of the macrophage (half way between the base and the top) showed a high degree of variability across the long axis of both nucleus and cytoplasm although macrophages exposed to HCNTs at $100 \mu \mathrm{g} / \mathrm{ml}$ had decreased Arp2 expression across much of the cytoplasm and nucleus compared to unexposed macrophages (Figure $4 \mathrm{c} \& 4 \mathrm{e}$ ). This pattern was also observed when Arp2 was measured across the short axis (Figure 4d \& 4f).

4a \& 4b: Arp2 fluorescence was measured across 11 slices for each macrophage. Slices were evenly distributed from the base to the top of the cells (a). Mean fluorescence intensity in the middle area of macrophages (slices 4-9) was significantly decreased in macrophages exposed to $100 \mu \mathrm{g} / \mathrm{ml}$ through slices 2-6. 4c-4f: Fluorescence was measured across the center of the cells at the mid line (slice 6). Both a long axis measurement which included the nucleus (c,e), and a short axis measurement which used equidistant 51 points from edge to edge. $4 \mathrm{~d} \& 4 \mathrm{f}$ were analyzed. Measurements across the cells showed the highest intensity along the cell membranes. Macrophages exposed to $100 \mu \mathrm{g} / \mathrm{ml}$ showed decreased fluorescence intensity in the mid cytoplasmic areas both along the long and short axis while the fluorescence intensity in macrophages exposed to $10 \mu \mathrm{g} /$ $\mathrm{ml}$ was similar to unexposed macrophages.

${ }^{*} \mathrm{p}<0.05$ (versus unexposed macrophages)

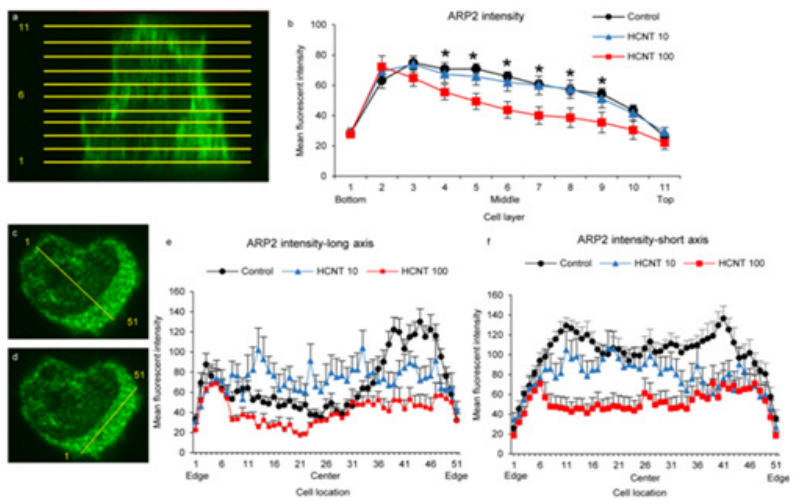

Figure 4 HCNTs impair Arp2 expression and limit its redistribution in RAW 264.7 macrophages.

\section{Intracellular accumulation of HCNTs attenuates the ability of alveolar macrophages to phagocytize $\mathbf{P}$. aeruginosa}

Our experiments with RAW 264.7 macrophages indicate that intracellular accumulation of HCNTs impaired the uptake of PAO1GFP. To confirm these results in an in vivo model, we examined whether 3 weeks of chronic HCNT exposure would lead to intracellular accumulation in alveolar macrophages and impairment of PAO1-GFP phagocytosis. Similar to what was observed with the RAW 264.7 macrophages, among alveolar macrophages which had internalized HCNTs, the majority had HCNTs occupying 1-20\% of the intracellular space, with decreasing percentage of macrophages showing increased HCNT accumulation (Figure 5a). A similar pattern to the experiments with the RAW 264.7 macrophages was observed regarding PAO1-GFP internalization. Significant inhibition of PAO1GFP internalization was observed in alveolar macrophages with an intracellular accumulation of $>20 \%$ compared to the alveolar macrophages from infected mice which did not receive HCNTs (Figure 5b). However, alveolar macrophages from HCNT exposed mice that did not take up any HCNTs had no significant difference in PAO1-GFP internalization from alveolar macrophages with a $1-20 \%$ intracellular burden. As HCNT accumulation exceeded $40 \%$ of intracellular area, internalization of PAO1-GFP was significantly inhibited.

5a: Following 3 weeks of repeated exposure to HCNT, the majority of alveolar macrophages harvested from mice have $1-20 \%$ of their intracellular space occupied by HCNTs. The accumulation of intracellular HCNTs was determined in 1000 macrophages. 5b: Increasing burden of HCNTs in alveolar macrophages reduced the uptake of PAO1-GFP. Red horizontal line indicates the percentage of PAO1-GFP positive alveolar macrophages recovered from control mice not exposed to HCNTs. $\mathrm{n}=4$ mice for each group and a minimum of 200 macrophages were evaluated from each animal.

${ }^{*} \mathrm{p}<0.05$ (versus unexposed alveolar macrophages); $\dagger \mathrm{p}<0.05$ (compared to HCNT-exposed alveolar macrophages with no evidence of HCNT accumulation). Error bars indicate standard error of the mean. 

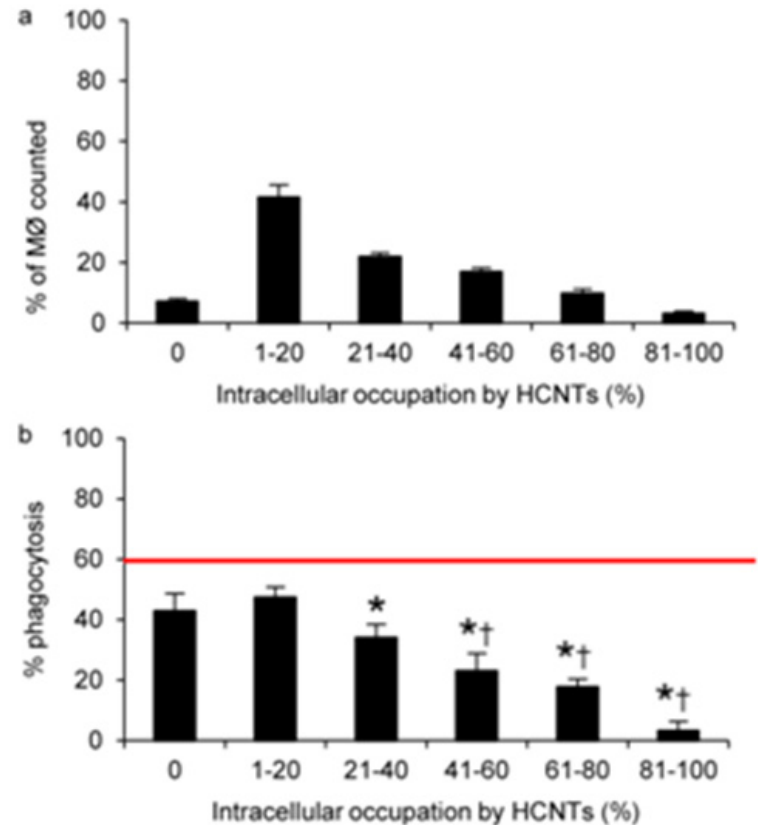

Figure 5 Intracellular accumulation of HCNTs decreases the uptake of $P$. aeruginosa in alveolar macrophages.

\section{Discussion}

The field of nanotoxicology has expanded since the early 2000's as the nanomaterial industry rapidly developed. With the respiratory system being a primary route of direct exposure, much work has focused on the direct impact of nanoparticles on pulmonary health. ${ }^{30-31}$ Multiple studies have demonstrated nanoparticles modulate cytokine release by leukocytes, but little work has involved known pulmonary pathogens. ${ }^{6,23,32}$ SWCNTs have been shown to inhibit the phagocytosis of apoptotic bodies and L. mono cytogenes. ${ }^{7,11}$ Studies comparing different types of nanotubes showed that both low and high doses of SWCNTs, as well as a high dose of MWCNTs, inhibited phagocytosis of latex beads equally well, while a low dose of MWCNTs was not as effective. ${ }^{9}$ Furthermore, larger diameter MWCNTs inhibited phagocytosis to a greater extent than those with smaller diameter, a pattern mirroring observed cytotoxicity in that study. ${ }^{10}$ In the same study, phagocytic function was also decreased with increasing concentrations of MWCNTs. Previously, we have shown that HCNTs inhibit internalization of $P$. aeruginosa by RAW 264.7 macrophages in a concentration dependent manner. ${ }^{8}$ In this study, we examine whether the degree of intracellular accumulation of HCNTs contributed to inhibition of phagocytosis of the respiratory pathogen $P$. aeruginosa. Our results indicated that inhibition of $P$. aeruginosa phagocytosis by macrophages was directly related to the amount of intracellular accumulation of HCNTs. Additionally, accumulated HCNTs inhibited F-actin polymerization and disrupted normal F-actin and Arp2 distribution in macrophages. Disruption of F-actin polymerization, and F-actin and Arp2 distribution, may explain how HCNTs interfere with the internalization of pathogens by macrophages.

Phagocytosis of particles and pathogens is an actin-dependent process which involves the binding of ligands to surface receptors followed by remodeling of the actin cytoskeleton. ${ }^{33}$ The binding of opsonins to cell surface receptors activates multiple downstream signals. ${ }^{23,27,31,32}$ which subsequently induce the remodeling of F-actin filaments by depolymerization and re polymerization at nucleation sites. ${ }^{14}$ Proper polymerization is essential for many cellular processes including motility, endocytosis, and the formation of phagocytic cups. ${ }^{13,34}$ The essential role of F-actin in phagocytosis has been demonstrated using the inhibitor cytochalasin $\mathrm{D}$, which blocks F-actin polymerization. ${ }^{35}$ The coordination of F-actin assembly to form the phagosome is directed by the Arp2/3 complex. ${ }^{14}$ Disruption of proper Arp2/3 complex localization will inhibit phagosome formation and prevent uptake of targets bound to receptors. ${ }^{16}$ Our study has demonstrated that uptake and accumulation of HCNTs alter F-actin and Arp2 distribution and expression in RAW 264.7 macrophages. These results, along with an independent study that demonstrated alterations in the cytoskeleton, including F-actin, by ultrafine particles which resulted in altered phagosome trafficking. ${ }^{17}$ suggests that HCNTs themselves may interfere with proper Arp2/3 complex localization, F-actin reorganization, and subsequent uptake of pathogens bound to macrophage receptors. Our data has shown that HCNTs altered F-actin and Arp2 intracellular distribution and inhibited F-actin polymerization. These suggest that HCNTs may impair and the formation of phagosome in response to attachment of pathogens and/or opsonins to phagocytic cell receptors.

Our results differ from Holt et al. ${ }^{20}$ who demonstrated that internalization of SWCNTs by HeLa cells causes disbursement of F-actin throughout the cell highlighted by intra-cytoplasmic spikes in actin intensity that were not observed in their control cells. In contrast, Snyder et al. ${ }^{22}$ demonstrated decreased intracellular F-actin intensity in MWCNT-exposed human bronchial epithelial cells, similar to what we observed in RAW 264.7 macrophages. The diversity of results illustrates how the biology of individual cell lines as well as the sizes and shapes of CNTs can differentially alter the function of cytoskeletal proteins. Furthermore, Holt et al. ${ }^{20}$ showed no inhibition of F-actin polymerization at concentrations of 0.5 and $5 \mu \mathrm{g} / \mathrm{ml} 20 .{ }^{20}$ while we demonstrated slight inhibition at $10 \mu \mathrm{g} / \mathrm{ml}$ and significant inhibition at $100 \mu \mathrm{g} / \mathrm{ml}$, indicating that the concentration of CNTs also is an important determinant in the intracellular accumulation of nanotubes and disruption of phagocytic function.

Our data does not preclude the possibility that other proteins are impacted by the presence of HCNTs. The Arp2/3 complex is composed of multiple proteins, which are involved in the nucleation of F-actin. The formation of the phagosome around receptor bound pathogens utilizes multiple proteins, which work in concert to polymerize $\mathrm{F}$-actin and depolymerize $\mathrm{F}$-actin around the phagocytic cups. These proteins are also important in the formation of stress fibers. For example, paxillin is a focal adhesion-associated signaling protein which acts as an adaptor involved in cytoskeleton assembly. ${ }^{36}$ Paxillin, along with vinculin. ${ }^{37}$ co-localizes with F-actin at the anchoring sites of focal adhesion complexes, and is involved in the regulation of cell motility. ${ }^{38}$ Studies have suggested that vinculin and paxillin are involved in immunoglobulin and complement mediated phagocytosis. $^{39-40}$ Importantly, paxillin becomes redistributed in cells treated with SWCNTs. ${ }^{20}$ Examination of paxillin and vinculin distribution as well as additional proteins associated with the Arp2/3 complex following exposure to CNTs would help elucidate their impact on F-actin distribution.

Our studies indicated that inhibiting the phagocytosis of $P$. aeruginosa required a relatively high intracellular accumulation of HCNTs. These results are similar to other studies, which demonstrated intracellular-related accumulation of nano-sized particles impairing alveolar macrophage function. Two studies which calculated accumulation of equal-sized round particles within macrophages showed that, when the uptake of uniform round particles exceeded $60 \%$ of the macrophage volume, phagocytic clearance of new particles was inhibited. ${ }^{41-42}$ These results were corroborated by Oberdorster et al. ${ }^{43}$ who showed how rat alveolar macrophages with a particle load of $\sim 60 \%$ of the normal volume failed to eliminate those particles from 
the lung. Furthermore, an independent study which demonstrated that exposure to a very low concentration of ultrafine particles actually enhanced the uptake of latex beads by J774.2 macrophages while high concentrations reduced uptake. ${ }^{37}$ Although they did not quantify the percentage of intracellular space occupied by the ultrafine particles, it is reasonable to conclude that the distribution of the macrophage population based on particle occupation would resemble our results at $10 \mu \mathrm{g} / \mathrm{ml}$, if not have an even greater number of macrophages in the $0-20 \%$ HCNT accumulation group, which showed no impairment in PAO1-GFP uptake.

One shortfall of our approach is that the RAW macrophages observed are a heterogeneous population with $0 \%, 1-20 \%, 21-40 \%$ intracellular HCNT burden, etc. which led to unequal counts in each group which, as stated above, may result in higher standard errors for smaller populations which could mask significant differences. Also, studies have suggested that macrophages which have taken up particles may release cytokine signals which impair unencumbered macrophage function, including migration and clearance of particles from the lung. ${ }^{44-45}$ Potentially, cytokines secreted by particleencumbered macrophages may impair the phagocytic function of unencumbered macrophages. Finally, separating macrophage populations based on intracellular HCNT burden prior to exposure to PAO1-GFP would be of value to compare phagocytic function but is not technically plausible.

Our results correlating inhibition of phagocytosis to the intracellular accumulation of CNTs expands on the complex relationship between particulate uptake and pulmonary innate immunity that has been observed by other investigators. Shvedova et al. ${ }^{7}$ demonstrated statistically similar inhibition of L. monocytogenes by alveolar macrophages despite using two different SWCNT exposure concentrations. Similarly, Jia et al. ${ }^{9}$ reported showed equal inhibition of the phagocytosis of latex beads following exposure to two different concentrations of SWCNTs, but that SWCNTs inhibited phagocytosis greater than MWCNTs, regardless of exposure concentration. Within the same report, the authors showed a concentration dependent inhibition of latex bead phagocytosis by MWCNTs. Moreover, a separate study reported a concentration dependent inhibition of the phagocytosis of latex beads by MWCNTs larger than $40 \mathrm{~nm}$ in diameter [10]. Collectively, these studies by us and others suggest that the type of CNTs internalized by human cells may differentially impact the biological machinery required to phagocytize targets.

\section{Conclusion}

In summary, we demonstrate that HCNTs can inhibit internalization of $P$. aeruginosa. Mechanistically, this inhibition is, in part, a result of the accumulation of HCNTs in the intracellular space. Intracellular accumulation of HCNTs disrupts the normal distribution of F-actin and Arp2, which are essential components of the phagocytic machinery, most likely through inhibition of F-actin polymerization. Furthermore, co- localization of F-actin and Arp2 in the cytoplasm distant from the cell membrane appears to be inhibited by a high concentration of HCNTs. Proteins with roles in F-actin anchoring and remodeling for movement and phagocytosis, including vinculin and paxillin, as well as other proteins in the Arp $2 / 3$ complex may also be affected by HCNT accumulation and are worthy of further examination.

\section{Acknowledgment}

None.

\section{Conflict of interest}

None.

\section{References}

1. Kayat J, Gajbhiye V, Tekade RK, Jain NK Pulmonary toxicity of carbon nanotubes: a systematic report. Nanomedicine. 2011;7(1):40-49.

2. Wang X, Xia T, Addo Ntim S et al. Dispersal state of multi walled carbon nanotubes elicits profibrogenic cellular responses that correlate with fibrogenesis biomarkers and fibrosis in the murine lung. ACS Nano. 2011;5(12):9772-9787.

3. Shvedova AA, Kisin ER, Mercer R et al. Un usual inflammatory and fibrogenic pulmonary responses to single-walled carbon nanotubes in mice. Am J Physiol Lung Cell Mol Physiol. 2005;289(5):698-708.

4. Ma-Hock L, Treumann S, Strauss V et al. Inhalation toxicity of multiwall carbon nanotubes in rats exposed for 3 months. Toxicological science.s 2009;112(2):468-481.

5. Herzog E, Byrne HJ, Casey A et al. SWCNT suppresses inflammatory mediator responses in human lung epithelium in vitro. Toxicol Appl Pharmacol. 2009;234(3):378-390.

6. Mitchell LA, Gao J, Wal RV et al. Pulmonary and systemic immune response to inhaled multi walled carbon nanotubes. Toxicol Sci. 2007;100(1):203-214.

7. Shvedova AA, Fabisiak JP, Kisin ER et al. Sequential exposure to carbon nanotubes and bacteria enhances pulmonary inflammation and infectivity. Am J Respir Cell Mol Biol. 2008;38(5):579-590.

8. Walling BE, Kuang Z, Hao Y et al. Helical Carbon Nanotubes Enhance the Early Immune Response and Inhibit MacrophageMediated Phagocytosis of Pseudomonas aeruginosa. PLoS One. 2013;8(11):80283-80283.

9. Jia G, Wang H, Yan L et al. Cytotoxicity of carbon nanomaterials: single-wall nanotube, multi-wall nanotube, and fullerene. Environ Sci Technol. 2005;39(5):1378-1383.

10. Wang X, Jia G, Wang $\mathrm{H}$ et al. Diameter effects on cytotoxicity of multiwalled carbon nanotubes. J Nanosci Nanotechnol. 2009;9(5):3025-3033.

11. Witasp E, Shvedova AA, Kagan VE et al. Single-walled carbon nanotubes impair human macrophage engulfment of apoptotic cell corpses. Inhal Toxicol Suppl. 2009;1:131-136.

12. Lavoie EG, Wangdi T, Kazmierczak BI Innate immune responses to Pseudomonas aeruginosa infection. Microb Infect. 2011;13(14-15):1133-1145.

13. May RC, Machesky LM Phagocytosis and the actin cytoskeleton. $J$ Cell Sci. 2001;114(6):1061-1077.

14. Millard TH, Sharp SJ, Machesky LM Signalling to actin assembly via the WASP (Wiskott-Aldrich syndrome protein)-family proteins and the Arp2/3 complex. Biochem J. 2004;380(1):1-17.

15. Cooper JA Effects of cytochalasin and phalloidin on actin. $J$ Cell Biol. 1987;105(4):1473-1478.

16. May RC, Caron E, Hall A, Machesky LM Involvement of the Arp2/3 complex in phagocytosis mediated by FcgammaR or CR3. Nat Cell Biol. 2000;2(4):246-248.

17. Moller W, Hofer $\mathrm{T}$, Ziesenis A et al. Ultrafine particles cause cytoskeletal dysfunctions in macrophages. Toxicol Appl Pharmacol. 2002;182(3):197-207.

18. Helfenstein M, Miragoli M, Rohr S et al. Effects of combustion-derived ultrafine particles and manufactured nanoparticles on heart cells in vitro. Toxicology. 2008;253(1-3):70-78.

19. Kaiser JP, Wick P, Manser P et al. Single walled carbon nanotubes (SWCNT) affect cell physiology and cell architecture. J Mater Sci Mater Med. 2008;19(4):1523-1527.

20. Holt BD, Short PA, Rape AD et al. Carbon nanotubes reorganize actin structures in cells and ex vivo. ACS Nano. 2010;4(8):4872-4878. 
21. Umemoto EY, Speck M, Shimoda LMN et al. Single-walled carbon nanotube exposure induces membrane rearrangement and suppression of receptor-mediated signalling pathways in model mast cells. Toxicol Lett. 2014;229(1):198-209.

22. Snyder RJ, Hussain S, Rice AB et al. Multi walled carbon nanotubes induce altered morphology and loss of barrier function in human bronchial epithelium at noncytotoxic doses. Int $J$ Nanomedicine. 2014;9:4093-4105.

23. Inoue K, Yanagisawa R, Koike E et al. Repeated pulmonary exposure to single-walled carbon nanotubes exacerbates allergic inflammation of the airway: Possible role of oxidative stress. Free Radic Biol Med. 2010;48(7):924-934.

24. Swedin L, Arrhigi R, Andersson-Willman B et al. Pulmonary exposure to single-walled carbon nanotubes does not affect the early immune response against Toxoplasma gondii. Part Fibre Toxicol. 2012;9:16.

25. Kuang Z, Hao Y, Hwang S et al. The Pseudomonas aeruginosa flagellum confers resistance to pulmonary surfactant protein-A by impacting the production of exoproteases through quorum-sensing. Mol Microbiol. 2011;79(5):1220-1235.

26. Zhang S, McCormack FX, Levesque RC et al. The Flagellum of Pseudomonas aeruginosa Is Required for Resistance to Clearance by Surfactant Protein A. PLoS One. 2007;2(6):564.

27. Muhlfeld C, Mayhew T, Gehr P et al. A novel quantitative method for analyzing the distributions of nanoparticles between different tissue and intracellular compartments. J Aerosol Med. 2007;20(4):395-407.

28. Tian F, Tian F, Prina-Mello A et al. A novel assay for the quantification of internalized nanoparticles in macrophages. Nanotoxicology. 2008;2(4):232-242.

29. Cooper JA, Pollard TD Methods to measure actin polymerization. Methods Enzymol. 1982;85(B) 182-210.

30. Shvedova AA, Kisin ER, Porter D et al. Mechanisms of pulmonary toxicity and medical applications of carbon nanotubes: Two faces of Janus? Pharmacol Ther. 1982;121(2):192-204.

31. Pacurari M, Qian Y, Porter DW et al. Multi-walled carbon nanotubeinduced gene expression in the mouse lung: association with lung pathology. Toxicol Appl Pharmacol. 2011;255(1):18-31.

32. Wang P, Nie X, Wang Y et al. Multiwall Carbon Nanotubes Mediate Macrophage Activation and Promote Pulmonary Fibrosis Through TGF- $\beta$ /Smad Signaling Pathway. Small. 2013;9(22):3799-3811.
33. Greenberg S, Grinstein S Phagocytosis and innate immunity. Curr Opin Immunol. 2002;14(1):136-145.

34. Kwiatkowska K, Sobota A Signaling pathways in phagocytosis. Bioessays. 1999;21(5):422-431.

35. Kaplan G Differences in the mode of phagocytosis with Fc and C3 receptors in macrophages. Scand J Immunol. 1977;6(8):797-807.

36. Turner CE Paxillin and focal adhesion signalling. Nat Cell Biol. 2000;2(12):231-236.

37. Renwick LC, Donaldson K, Clouter A Impairment of Alveolar Macrophage Phagocytosis by Ultrafine Particles. Toxicol Appl Pharmacol. 2001;172(2):119-127.

38. Liu S, Thomas SM, Woodside DG, Rose DM et al. Binding of paxillin to alpha 4 integrins modifies integrin-dependent biological responses. Nature. 1999;402(6762):676-681.

39. Allen LA, Aderem A Molecular definition of distinct cytoskeletal structures involved in complement- and Fc receptor-mediated phagocytosis in macrophages. J Exp Med. 1996;184(2):627-637.

40. Greenberg S, Chang P, Silverstein SC Tyrosine phosphorylation of the gamma subunit of Fc gamma receptors, p72syk, and paxillin during Fc receptor-mediated phagocytosis in macrophages. $J$ Biol Chem. 1994;269(5):3897-3902.

41. Morrow PE Possible mechanisms to explain dust overloading of the lungs. Fundam Appl Toxicol. 1988;10(3):369-384.

42. Lehnert BE, Ortiz JB, London JE et al. Migratory behaviors of alveolar macrophages during the alveolar clearance of light to heavy burdens of particles. Exp Lung Res. 1990;16(5):451-479.

43. Oberdorster G, Ferin J, Morrow PE Volumetric loading of alveolar macrophages (AM): a possible basis for diminished AM-mediated particle clearance. Exp Lung Res. 1992;18(1):87-104.

44. Bellmann B, Muhle $\mathrm{H}$, Creutzenberg $\mathrm{O}$ et al. Lung clearance and retention of toner, utilizing a tracer technique, during chronic inhalation exposure in rats. Fundam Appl Toxicol. 1991;17(2):300-313.

45. Morrow PE Dust overloading of the lungs: Update and appraisal. Toxicol Appl Pharmacol. 1992;113(1):1-12. 